\title{
Neuroendocrine carcinoma of esophageal and gastric cardia: clinicopathologic and immunohistochemistry study of $\mathbf{8 0}$ cases
}

\author{
Liangli Hong ${ }^{1, *}$, Ying Zhang ${ }^{2, *}$ and Zhaoyong Liu ${ }^{3}$ \\ ${ }^{1}$ Department of Pathology, First Affiliated Hospital of Shantou University Medical College, Shantou, Guangdong, China \\ ${ }^{2}$ Department of Pathology, Shantou University Medical College, Shantou, Guangdong, China \\ ${ }^{3}$ Department of Orthopedics, First Affiliated Hospital of Shantou University Medical College, Shantou, Guangdong, China \\ *These authors contributed equally to this work
}

Correspondence to: Zhaoyong Liu, email: langtian07@hotmail.com

Ying Zhang, email: yzhangmimazi@stu.edu.cn

Keywords: neuroendocrine tumor; esophageal; gastric cardia; prognostic

Received: June 21, $2017 \quad$ Accepted: November 26, $2017 \quad$ Published: December 22, 2017

Copyright: Hong et al. This is an open-access article distributed under the terms of the Creative Commons Attribution License 3.0 (CC BY 3.0), which permits unrestricted use, distribution, and reproduction in any medium, provided the original author and source are credited.

\section{ABSTRACT}

Neuroendocrine carcinoma (NEC) of the esophagus and gastric cardia is a rare tumor, and the Chaoshan region has one of the highest incidences of esophageal and gastric cardia cancer (GCC) worldwide. The aim of this study was to characterize the clinicopathologic features of esophageal NEC $(n=67)$ and gastric cardia NEC $(n=13)$ cases identified over a 9-year period in the Chaoshan region. Esophageal NECs were either purely NEC $(n=47)$ or mixed with squamous cell carcinoma or adenocarcinoma $(n=20)$. For GCC; pure NEC was found in 5 cases, whereas 8 cases were mixed with adenocarcinomas. The majority of esophageal and gastric cardia NECs was of the small cell type, and 24/67 esophageal and 5/13 gastric cardia patients were found with lymph node metastasis. Immunohistochemistry was performed in all cases, and positive staining for synaptophysin (Syn) was found for all cases, with half the esophageal NEC cases being also chromogranin A ( $\mathrm{CgA}$ )-positive. In the multivariate Cox regression model, lymph node and further metastasis were independent prognostic factors for esophageal NEC. Our study revealed the clinicopathological features of esophageal and gastric cardia NECs in the Chaoshan region and found mixed NECs patients may have a better prognosis than pure NECs patients, which may provide therapeutic clue for treating this rare tumor.

\section{INTRODUCTION}

Neuroendocrine tumors(NETs) are defined as neoplasms that exhibit neuroendocrine phenotypes, such as production of neuropeptides, large dense-core secretory vesicles, and a lack of neural structures [1]. According to the World Health Organization (WHO) classification scheme in 2010, NETs can bedivided into three main categories: well-differentiated (low-grade), which is benign, mediumdifferentiated (intermediate-grade) with low-grade malignant behavior, and poorly-differentiated (high grade), which can itself be divided into large cell and small cell neuroendocrine carcinomas (NECs) [2]. Additionally, adenocarcinomas that contain neuroendocrine cells (exceeding at least $30 \%$ of all tumor cells) mixed with non-endocrine components (usually adenocarcinoma-like structures) are classified asmixed adenoneuroendocrine carcinomas (MANECs) [3]. NEC, a malignant type of NET, has been reported in pancreatic, breast, lung and bronchial cancers [4-6].

Esophageal NEC is a rare esophageal cancer consisting of neuroendocrine components, and comprises $0.5-5.9 \%$ of all esophageal malignancies. During the past 20 years, a limited number of cases has been reported in the literature.NECs appears to confer a worse prognosis than other malignant esophageal cancers, probably due to its poor differentiation and lymphovascular invasion. However, the clinicopathologic characteristics of NECs have not been fully described. 
The gastric cardia is a very narrow area between the esophagus and gastric fundus. This specialized mucosa contains a mixture of squamous and glandular epithelium [7]. The gastric cardia mucosa is gastric in origin, and strong evidence shows that the gastric cardia contains numerous endocrine cells, which are mainly located on the surfaces of adjacent mucosal or parietal cells [8]. Owing to its unique location and biological features, gastric cardia cancer is mainly divided into two distinct types, one type being located in the distal stomach as a consequence of atrophic gastritis, and the other type resembling esophageal adenocarcinoma that may be a consequence of short-segment gastro-esophageal reflux disease [7]. However, NECs in the gastric cardia are rare,representing only $0.04-7.6 \%$ of the gastrointestinal NECs that have been reported [9].

The Chaoshan area has the highest incidence of esophageal cancer and gastric cardia cancer worldwide, with the age-standardized incidence rates for esophageal and gastric cardia cancer in the Chaoshan area respectively being $100 / 100,000$ and $34.81 / 100,000$, which are much higher than the worldwide incidence $(7 / 100,000)$, suggesting unique environmental/genetic factors involved in esophageal and gastric cardia cancer in the Chaoshan area $[10,11]$.In the present study, we collected surgical specimens of esophageal and gastric cardia NEC patients who underwent esophagectomy with regional lymphadenectomy in Chaoshan Hospital from January 2007 to December 2016, and investigated the clinicopathologic and immunohistochemical data following tumor definition based on the WHO classification of NECs.

\section{RESULTS}

All medical records of esophageal cancer and gastric cardia cancer patientsin the Chaoshan region from 2007 to 2016 were searched. Among 3105 esophageal cancer and 823 gastric cardia cancer patients, 67 (2.1\%) esophageal NEC and $13(1.6 \%)$ gastric cardia NEC cases were diagnosed and selected for further investigation.

\section{Clinical characteristics}

The clinical characteristics of 67 esophageal patients from the Chaoshan area are summarized in Table 1. Of the 67 esophageal NEC patients, 47 were men and 20 were women, with the ratio of men to women being $2: 1$, and the average patient age was 58.5 years (ranging from 44 to 75$)$. Overall, 54/67 (80.5\%) of the NEC patients had dysphagia for over 20 days to 3months, and 11/67 (16.4\%) patients felt pain in the chest. Gastroscopy, esophagoscopy or gastroesophageal CT was performed on 65 patients before pathological diagnosis. Just like esophageal squamous cell carcinoma, the gross appearances of NECs were mainly divided into four types: ulcerated (24/67), fungating (18/67), medullar (20/67) and stenotic(5/67). On the tumor surface, necrosis was found in $88 \%$ of the cases, and blood was seen in $20(29.8 \%)$ cases. The tumor size was $3.5 \mathrm{~cm}$ on average, and ranged from 0.2 to $8 \mathrm{~cm}$. For esophageal NEC, the majority of tumors (40/67) were located in the middle segment of the esophagus, 13/67 in the upper esophagus, and 14/67 in the distal esophagus.

The detailed information of gastric cardia NEC patients $(n=13)$ is listed in Table 2. The age of the gastric cardia NEC patients ranged from 29 to 78 , with 8 of the 13 gastric cardia NEC patients presenting with symptoms of gastroesophageal reflux, and 5/13 presenting vomit or blood in the stool. The gastric cardia NECs were all located within $2 \mathrm{~cm}$ from the gastroesophageal junction. The majority of tumors were ulcerated (9/13) and had sizes ranging from $0.5-5.5 \mathrm{~cm}$.

\section{Pathology stage}

Based on the 8th edition of the AJCC TNM staging system for ESCC, the tumor stages of the esophageal NECs are shown in Table 3. A significant difference was observed in tumor differentiation and clinical stage between pure NEC and mixed NEC groups.The pathological features of gastric cardia NEC patients are listed in Table 2. Lymph node metastasis was found in $5 / 13$ cases, with metastasis of the regional lymph node being the most common. The majority of cases (11/13) were stage II-III, and none of the cases displayed distant metastasis.

\section{Histopathologic features}

As shown in Table 1, under high-power observation, the majority (94\%) of esophageal NEC cells displayed a round/ovoid, spindle or anaplastic shape, indicating tumorscould be classified as small cell neuroendocrine carcinomas (SNECs) (Figure 1A). In contrast, large cell neuroendocrine carcinoma cells(LNECs) were large with irregular, bizarre nuclei and ample eosinophilic cytoplasm (Figure 1B). Esophageal SNEC was present in $63(94.0 \%)$ patients, and $4(6 \%)$ patients had LNEC; 20 (29.9\%) patients had mixed NEC, and $47(70.1 \%)$ patients had pure NEC. Two (10\%) patients had mixed NEC and adenocarcinoma, and $18(90 \%)$ patients had mixed NEC andsquamous carcinoma, including 17 (94.4\%) patients with SNEC and $1(0.6 \%)$ with LNEC (Figure 1C, 1D).

Table 1 compares the clinicopathologic features of SNEC vs. LNEC, and pure NEC vs. mixed NEC. No significant difference was noted in age, sex, gross type, tumor location or tumor size between these groups. Microscopic observation revealed that the majority of NECs exhibited a nodular growth pattern with a clear border. The tumor border was infiltrated with capillaries and inflammatory cells, such as lymphocytes and plasmacytes, and two tumors showed rare lymphoid 


\begin{tabular}{|c|c|c|c|c|c|c|c|}
\hline \multirow{2}{*}{$\begin{array}{c}\text { Pathologic } \\
\text { Characteristics }\end{array}$} & \multirow[t]{2}{*}{ Total } & \multirow[b]{2}{*}{ SCNC } & \multirow[b]{2}{*}{ LCNC } & \multirow[b]{2}{*}{$P$-value } & \multirow[b]{2}{*}{ Mixed NEC } & \multirow[b]{2}{*}{ Pure NEC } & \multirow[b]{2}{*}{$P$-value } \\
\hline & & & & & & & \\
\hline \multicolumn{8}{|l|}{ Age } \\
\hline$>\mathbf{5 9}$ & 53 & 51 & 2 & 0.19 & 15 & 38 & 0.74 \\
\hline$\leq 58$ & 14 & 12 & 2 & & 5 & 9 & \\
\hline \multicolumn{8}{|l|}{ Location } \\
\hline Upper & 13 & 12 & 1 & 0.91 & 1 & 12 & 0.06 \\
\hline Middle & 40 & 38 & 2 & & 12 & 28 & \\
\hline Distal & 14 & 13 & 1 & & 7 & 7 & \\
\hline \multicolumn{8}{|l|}{ Tumor size } \\
\hline$<3.5 \mathrm{~cm}$ & 34 & 33 & 1 & 0.36 & 9 & 25 & 0.60 \\
\hline$>3.5 \mathrm{~cm}$ & 33 & 30 & 3 & & 11 & 22 & \\
\hline \multicolumn{8}{|l|}{ Gross appearance } \\
\hline Ulcerated & 24 & 23 & 1 & 0.79 & 5 & 19 & 0.53 \\
\hline Fungating & 18 & 17 & 1 & & 5 & 13 & \\
\hline Medullary & 20 & 18 & 2 & & 8 & 12 & \\
\hline Stenosis & 5 & 5 & 0 & & 2 & 3 & \\
\hline \multicolumn{8}{|l|}{ Gender } \\
\hline Male & 47 & 44 & 3 & 1 & 13 & 34 & 0.57 \\
\hline Female & 20 & 19 & 1 & & 7 & 13 & \\
\hline
\end{tabular}

follicles (Figure 2A). However, some tumors displayed an invasive pattern in that the tumor cells broke the tumor boundary to mix with other types of cancer cells or normal cells (Figure 2B). Tumor stroma was rich in vasculature in most $(83.6 \%)$ cases with marked proliferation of capillaries and small venules both being most prominent in the superficial portion of the tumor, and with submucosal and subserosal regions surrounding the tumor (Figure $2 \mathrm{C}, 2 \mathrm{D})$. In all esophageal NEC tumors, intratumoral small lymphocytic infiltration was characteristically absent or minimal. Calcification, osteoid metaplasia, or granuloma was not observed in any cases. Tumor metastasis to regional nodes (24/24) and abdominal celiac lymph nodes (11/24) was substantial(Figure 2E, 2F). The data further showed that metastasis affected the juxtaesophageal lymph node (11/24), trachea carina (8/24), left supraclavicular lymph nodes $(7 / 24)$, left gastric arteria lymph node (4/24), left recurrent laryngeal nerve lymph node (3/24) and stomach cardia lymph node (2/24).

In gastric cardia NEC patients, all NEC swere of the small type, with $8 / 13$ patients showing mixed NEC with adenocarcinoma. In particular, two NEC patients had a mixed NEC with mucinous adenocarcinoma. The border between these two types of cancer was clear and
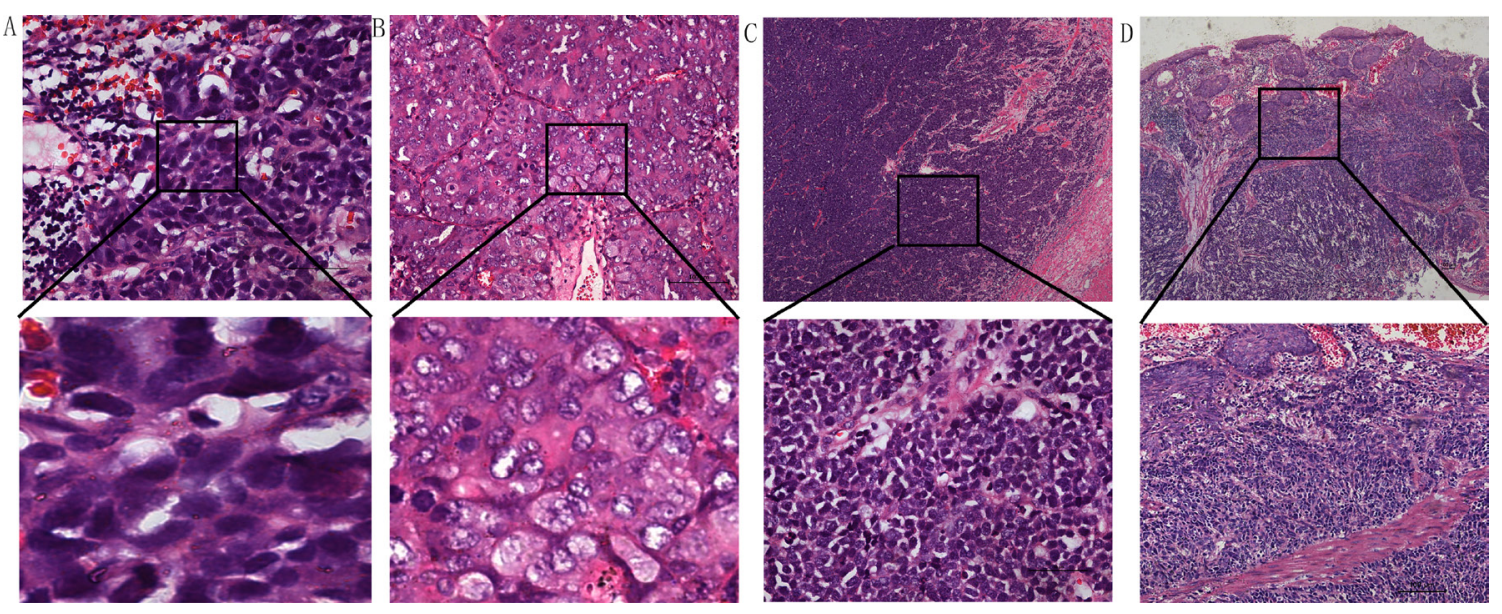

Figure 1: (A) Small cell esophageal NEC showing oat-like features. (B) Large cell esophageal NEC. (C) Pure esophageal NEC. (D) Mixture of NECs and squamous cell carcinoma. Hematoxylin-eosin stain in (A-D).(Scale bar: $100 \mu \mathrm{m})$ 
Table 2: Clinicopathological data on patients diagnosed with gastric cardia NEC

\begin{tabular}{|c|c|c|c|c|c|c|c|c|c|c|}
\hline NO. & Gender & Age & $\begin{array}{c}\text { Tumor } \\
\text { size }\end{array}$ & Gross type & Symptom & $\begin{array}{l}\text { Histopathology } \\
\text { (NECs with) }\end{array}$ & Stage & $\begin{array}{c}\text { IHC-positive } \\
\text { staining }\end{array}$ & $\begin{array}{l}\text { Treatment } \\
\text { (Surgery) }\end{array}$ & $\begin{array}{c}\text { Follow- } \\
\text { up }\end{array}$ \\
\hline 1 & M & 68 & $2 \mathrm{~cm}$ & ulcerating & GR & $\mathrm{AD}$ & $\begin{array}{l}\text { T2N0M0 } \\
\text { (I) }\end{array}$ & Syn, CK & & NA \\
\hline 2 & M & 69 & $4.5 \mathrm{~cm}$ & fungal & VB & $\mathrm{AD}$ & $\begin{array}{l}\text { T3N0M0 } \\
\text { (II) }\end{array}$ & $\begin{array}{l}\text { Syn, CgA, } \\
\text { CEA }\end{array}$ & & NA \\
\hline 3 & M & 65 & $0.5 \mathrm{~cm}$ & fungal & VB & $\mathrm{AD}$ & $\begin{array}{l}\text { T3N0M0 } \\
\text { (II) }\end{array}$ & Syn, CgA & $\mathrm{CT}$ & NA \\
\hline 4 & M & 63 & $2.3 \mathrm{~cm}$ & ulcerating & GR & MAD & $\begin{array}{l}\text { T4N3M0 } \\
\text { (III) }\end{array}$ & Syn, CK8/18 & & 5 \\
\hline 5 & M & 58 & $1.7 \mathrm{~cm}$ & ulcerating & VB & $\mathrm{AD}$ & $\begin{array}{l}\text { T4N2M0 } \\
\text { (III) }\end{array}$ & Syn, CgA & & 10 \\
\hline 6 & M & 78 & $0.8 \mathrm{~cm}$ & ulcerating & GR & Pure & $\begin{array}{l}\text { T3N1M0 } \\
\text { (II) }\end{array}$ & Syn, CD56 & $\mathrm{CT}$ & NA \\
\hline 7 & M & 44 & $1.1 \mathrm{~cm}$ & ulcerating & GR & Pure & $\begin{array}{l}\text { T3N0M0 } \\
\text { (II) }\end{array}$ & Syn, CD56 & & NA \\
\hline 8 & M & 65 & $3.5 \mathrm{~cm}$ & ulcerating & VB & $\mathrm{AD}$ & $\begin{array}{l}\text { T4N0M0 } \\
\text { (III) }\end{array}$ & Syn, CK8/18 & & NA \\
\hline 9 & M & 65 & $1.3 \mathrm{~cm}$ & ulcerating & GR & Pure & $\begin{array}{l}\text { T3N1M0 } \\
\text { (II) }\end{array}$ & $\begin{array}{l}\text { Syn, CgA, } \\
\text { CK }\end{array}$ & & 18 \\
\hline 10 & M & 78 & $0.5 \mathrm{~cm}$ & fungal & GR & Pure & $\begin{array}{l}\text { T4N0M0 } \\
\text { (III) }\end{array}$ & $\begin{array}{l}\text { Syn, CgA, } \\
\text { CK }\end{array}$ & CT & NA \\
\hline 11 & $\mathrm{~F}$ & 69 & $0.9 \mathrm{~cm}$ & fungal & GR & MAD & $\begin{array}{l}\text { T3N1M0 } \\
\text { (II) }\end{array}$ & Syn, CgA & & NA \\
\hline 12 & F & 63 & $3.4 \mathrm{~cm}$ & ulcerating & VB & Pure & $\begin{array}{l}\text { T4N0M0 } \\
\text { (III) }\end{array}$ & Syn, CD56 & $\mathrm{CT}$ & 10 \\
\hline 13 & $\mathrm{~F}$ & 29 & $2.2 \mathrm{~cm}$ & ulcerating & GR & $\mathrm{AD}$ & $\begin{array}{l}\mathrm{T} 2 \mathrm{~N} 0 \mathrm{M} 0 \\
\text { (I) }\end{array}$ & Syn, CK & $\mathrm{CT}+\mathrm{RT}$ & Alive \\
\hline
\end{tabular}

M: male; F: female; GR: gastroesophageal reflux, VB: vomit or blood in the stool CT: chemotherapy; RT: radiotherapy

wide, and contained infiltrating inflammatory cells (Figure 3A, 3B). In some pure NEC cases, the cancer cells had a tendency to break the boundary and invade into the surrounding normal tissues (Figure 3C, 3D).

\section{Immunohistochemical studies}

Syn, CgA, CD56, S-100, et al were used to detect the NECs (Figure 4). Esophageal neoplastic cells exhibited strong, diffuse immunoreactivity to Syn in all cases and to NSE in $9 / 10$ cases (Table 4). Strong immunoreactivity was also observed for CD56 in 18/20 of the cases, and to $\mathrm{CgA}$ in 30/54 cases, with variable proportions. Neoplastic cells in 7/14 of the cases were also immunoreactive for S-100. Positive nuclear TTF-1 immunoreactivity was demonstrated in $6 / 11$ of the cases. In the mixed NEC patient samples, 7/20 tumors were immunoreactive to p63 antibody and 11/15 cases were positive for CK. Evident immunoreactivity to Ki67 was substantial (ranging from $30 \%-90 \%$ of the NEC cells). There was no significant difference in any of the immunoreactivities between large cell and small cell types (Table 4).

Immunohistochemistry of gastric cardia NEC tumors is listed in Table 2. Syn was positive in 7/7 cases, and $6 / 6$ cases were positive for $\mathrm{CgA}$. Immunoreactivity was also observed for CK5/6 in $4 / 5$ of the cases and the positive incidence of Ki67 ranged from less than $30 \%$ to $70 \%$.

\section{Patient survival}

Survival data was available for $34 / 67$ patients, and the overall median follow-up period was 23.0 months, within which 17 patients died. The 1-, 3- and 5 -year overall survival was $88.2 \%, 55.9 \%$ and $29.5 \%$, respectively, with no significant difference in the survival of NEC and ESCC patients.

Univariate and multivariate analyses were performed to assess the relationship between clinicopathological features and prognosis (Table 5). In univariate analysis, lymph node metastasis and distant metastasis showed statistical difference. Survival of patients with lymph node metastasis was significantly shorter ( 24.5 vs. 10.7 months, $P=0.02$ ). Patients with distant metastasis revealed a shorter survival time than patients without (11.6 vs. 33.8 months, respectively, $P=$ $0.01)$. However, univariate analysis showed there were no significant differences in survival time with regard to 
Table 3: Pathological stages of esophageal NEC patients

\begin{tabular}{|c|c|c|c|c|c|c|c|}
\hline \multirow{2}{*}{ Pathologic Characteristics } & \multirow[t]{2}{*}{ Total } & \multicolumn{2}{|c|}{ Classification } & \multirow[b]{2}{*}{$p$} & \multirow[b]{2}{*}{ Mixed NEC } & \multirow[b]{2}{*}{ Pure NEC } & \multirow[b]{2}{*}{$P$} \\
\hline & & SCNC & LCNC & & & & \\
\hline \multicolumn{8}{|l|}{ Differentiation (G) } \\
\hline Well（G1） & 0 & 0 & 0 & & 0 & 0 & \\
\hline Moderate (G2) & 19 & 19 & 0 & 0.57 & 2 & 17 & 0.04 \\
\hline Poor (G3) & 48 & 44 & 4 & & 18 & 30 & \\
\hline \multicolumn{8}{|l|}{ Invasion } \\
\hline $\mathrm{T} 1$ & 3 & 3 & 0 & 0.50 & 2 & 1 & 0.26 \\
\hline $\mathrm{T} 2$ & 14 & 13 & 1 & & 3 & 11 & \\
\hline $\mathrm{T} 3$ & 30 & 27 & 3 & & 7 & 23 & \\
\hline $\mathrm{T} 4$ & 20 & 20 & 0 & & 8 & 12 & \\
\hline \multicolumn{8}{|l|}{ Lymph node metastasis } \\
\hline N0 & 43 & 40 & 3 & 0.57 & 17 & 26 & 0.07 \\
\hline N1 & 14 & 14 & 0 & & 1 & 13 & \\
\hline N2 & 7 & 6 & 1 & & 1 & 6 & \\
\hline N3 & 3 & 3 & 0 & & 1 & 2 & \\
\hline \multicolumn{8}{|l|}{ Distal Metastasis } \\
\hline M0 & 58 & 54 & 4 & 1 & 15 & 43 & 0.11 \\
\hline M1 & 9 & 9 & 0 & & 5 & 4 & \\
\hline \multicolumn{8}{|l|}{ Clinical Stage } \\
\hline I & 17 & 17 & 0 & 0.22 & 8 & 9 & $0.02^{*}$ \\
\hline II & 18 & 17 & 1 & & 3 & 15 & \\
\hline III & 21 & 18 & 3 & & 3 & 18 & \\
\hline IV & 11 & 11 & 0 & & 6 & 5 & \\
\hline
\end{tabular}

gender $(P=0.74)$, age $(P=0.79)$, tumor site $(P=0.62)$, gross tumor type $(P=0.44)$, tumor invasion $(P=0.26)$, tumor differentiation $(P=0.21)$, tumor size $(P=0.98)$ and having undergone or not undergone chemotherapy $(P=$ 0.48). Furthermore, in multivariate analysis conducted using the Cox proportional hazards mode, lymph node and distant metastasis showed significant differences $(P=$ $0.01)$, with patients with lymph node or distant metastasis having a shorter median survival time.

\section{DISCUSSION}

In this paper, we characterize the clinicopathological features of neuroendocrine carcinoma of the esophagus and gastric cardia, a relatively rare malignant tumor, in the Chaoshan region. The characteristics of esophageal NECs are as follows: 1 . tumors are predominantly located in the center of the esophagus; 2 . the small cell type comprises the main histological classification; 3 . gross appearance is mainly of the ulcerative type; 4 . half of the patients have lymph node metastasis and the juxta-esophageal lymph node is the most frequently affected; and 5. patients who have distal metastasis or lymph node metastasis have a worse clinical outcome. Gastric cardia NEC is primarily mixed with adenocarcinoma, the ulcerative type is the most common gross appearance, and the regional lymph node is the most frequent metastatic site.

NEC is defined as a high grade neuroendocrine carcinoma (G3), data regarding the prevalence of esophageal and gastric cardia NEC among patients is limited. Lyomasa et al. reported that esophageal NECs comprise approximately $1-2 \%$ of all esophageal neoplasms in China [13]. The current study indicates that the incidence of esophageal NECs has been approximately $2.1 \%$ of all esophageal cancers in Chaoshan over the past 6 years. Esophageal NEC closely correlates with Barrett's esophagus [14]. However, the origin of this tumor has not been clearly demonstrated. Some reports indicate that NEC originates from Kulchisky cells in the esophageal epithelium, which are amine precursor uptake and decarboxylation (APUD) cells, since theyhave APUD characteristics [15]. However, other reports indicate esophageal NECs originate from pluripotent stem cells that can differentiate into various epithelial cells. Our finding supports this concept since NECs are always mixed with other types of cancer, e.g. SCC or adenocarcinoma, especially in the gastric cardia.

In general, esophageal cancers are often present as an intraluminal exophytic mass, which results in an early 
Table 4: Immunostaining for CgA, Syn, CD56, NSE, S100, P63, TTF-1, and CK

\begin{tabular}{lcccc}
\hline \multirow{2}{*}{ Antigen } & Total & \multicolumn{3}{c}{ Classification } \\
\cline { 3 - 5 } & & SCNC & LCNC & 1 \\
\hline CgA & $30 / 56$ & $28 / 53$ & $2 / 3$ & 1 \\
Syn & $67 / 67$ & $63 / 63$ & $4 / 4$ & 0.28 \\
CD56 & $18 / 20$ & $16 / 17$ & $2 / 3$ & 1 \\
NSE & $9 / 10$ & $9 / 10$ & 0 & 1 \\
S100 & $7 / 14$ & $6 / 13$ & $1 / 2$ & 0.35 \\
P63 & $7 / 20$ & $6 / 19$ & $1 / 1$ & 1 \\
TTF-1 & $6 / 11$ & $6 / 11$ & 0 \\
CK5/6 & $11 / 15$ & $8 / 11$ & $3 / 4$ & 1 \\
\hline
\end{tabular}

onset of symptoms and allows for earlier diagnosis and treatment. Although esophageal NECs have a tendency for endoluminal growth, this does not mean that this type of tumor has limited malignant potential. We found that the major gross type of NEC is ulcerated, with the necrosis and hemorrhage on the surface of the tumor. In the majority of cases, cancer cells have infiltrated into the muscular layer as far as the serosa layer, indicating that NEC cells can be aggressively invasive.

The major pathological type of esophageal NEC involves small cell NEC, with most esophageal NECs are pure NECs. We only observed two NECs mixed with adenocarcinoma and others mixed with SCC. In those two cases, the patients had a history of gastrointestinal reflux. Our findings are consistent with Huang et al., who described 42 patients suffering from high-grade neuroendocrine carcinoma of the esophagus in China and found that most (88\%) NECs were of the small cell type [13]. However, Maru et al. reviewed 40 cases of esophageal NEC reported in the USA, in which 27/40 cases were of the large cell type [16]. In contrast, our data show that an overwhelming number of NECs in the Chaoshan population is of the small cell type, suggesting that NEC in the Chinese population represents a unique esophageal neoplasm. One possible explanation of this difference is the geographical variation and population genetics.

Our group of esophageal NEC patients, in the Chaoshan region, were in the late stages, with 32 of the
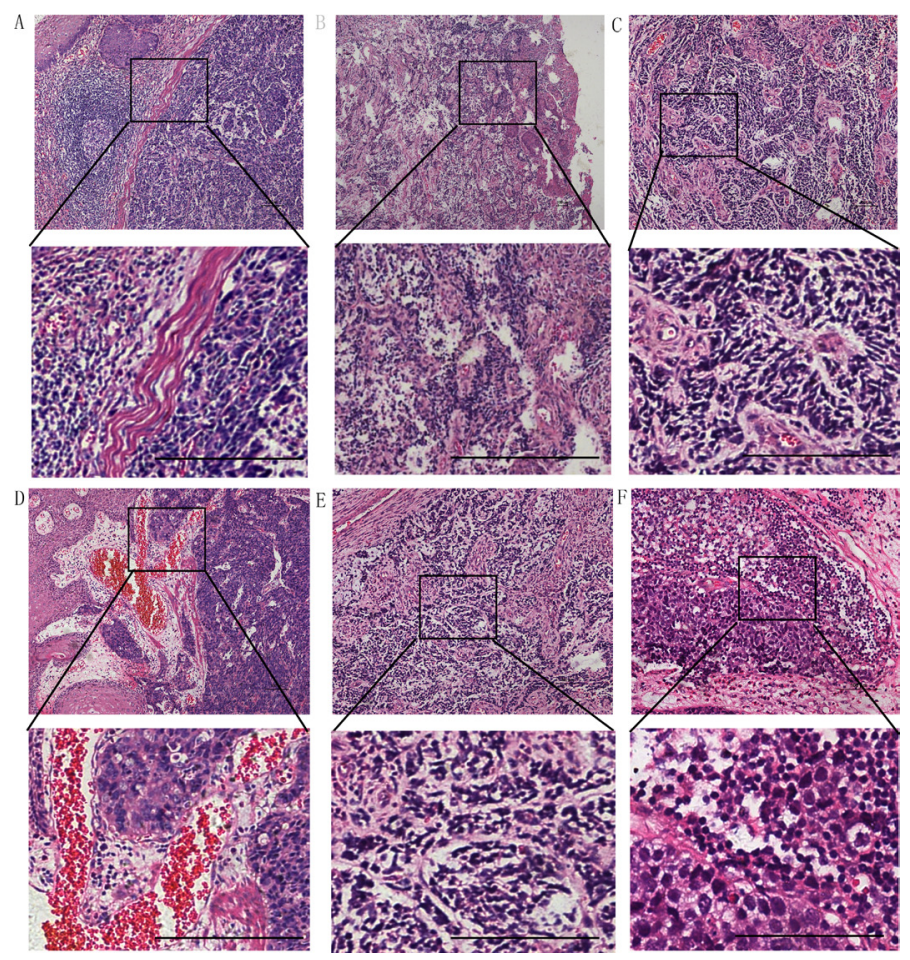

Figure 2: (A) The boundary between NEC and squamous cell carcinoma is clear and lymphocytes infiltrating into lymphoid follicles. (B) NEC cells were mixed with esophageal squamous carcinoma cells without a border. Tumor stroma was infused with blood vessels in most cases with capillaries (C) and venules (D). Lymph node metastasis could be found in 24 cases (E, F). Hematoxylin-eosin stain in A-D. (Scale bar: $100 \mu \mathrm{m}$ ) 
Table 5: Univariate and multivariate analysis of prognostic factors for esophageal NEC

\begin{tabular}{|c|c|c|c|c|}
\hline & Univariate & & Multivariate & \\
\hline & HR $(95 \% C I)$ & $P$-value & HR $(95 \% C I)$ & $P$-value \\
\hline Age, years $(<58$ vs. $\geq 58)$ & $1.3(0.1-15.2)$ & 0.79 & & \\
\hline Gender (male vs. female) & $0.75(0.39-1.44)$ & 0.74 & & \\
\hline Tumor site (middle vs. others) & $1.34(0.25-7.89)$ & 0.62 & & \\
\hline Tumor size $(>5 \mathrm{~cm}$ vs. $<5 \mathrm{~cm})$ & $1.05(0.05-23.2)$ & 0.98 & & \\
\hline Gross type (variable) & $0.33(0.75-2.66)$ & 0.44 & & \\
\hline Depth of invasion (continuous variable) & $0.51(0.05-5.17)$ & 0.26 & & \\
\hline Lymph node metastasis (yes vs. no) & $14.74(1.26-172.4)$ & 0.02 & $6.86(2.22-21.21)$ & 0.01 \\
\hline Differentiation (continuous variable) & $0.11(0.01-3.32)$ & 0.21 & & \\
\hline Treatment (CT vs. no CT) & $0.37(2.96-6.75)$ & 0.48 & & \\
\hline Distant Metastasis (yes vs. no) & $33.1(2.02-543.5)$ & 0.01 & $15.6(3.14-77.62)$ & 0.01 \\
\hline
\end{tabular}

cases being in stage III-IV, 24/67 of patients presenting with metastasis, mainly of the regional lymph nodes, and 9 patients with distant metastasis. A high frequency of lymph node metastases and distant metastases has been reported for esophageal NECs. Recently, Nayal et al. reported 11 cases with small cell carcinoma of the esophagus in India, with $8 / 11$ having distant metastases, the liver and lung being common sites $[17,18]$. Huang et al. found that half of esophageal NEC cases have lymphovascular (50\%) and lymph node metastasis [13]. In our study, all patients had surgical resection and lymphadenectomy. The literature reports that neuroendocrine carcinoma of the esophagus has lymph node metastasis in the very early stages, and that the ratio of lymph node metastasis is higher than for esophageal squamous cell carcinoma [15]. The importance of lymphadenectomy should be highlighted as much as possible, since this is a possible approach to cure the disease. Patients with lymphadenectomyhave good prognosis [15].

Although it is not essential to do immunohistochemistry to diagnose NEC tumors, IHC

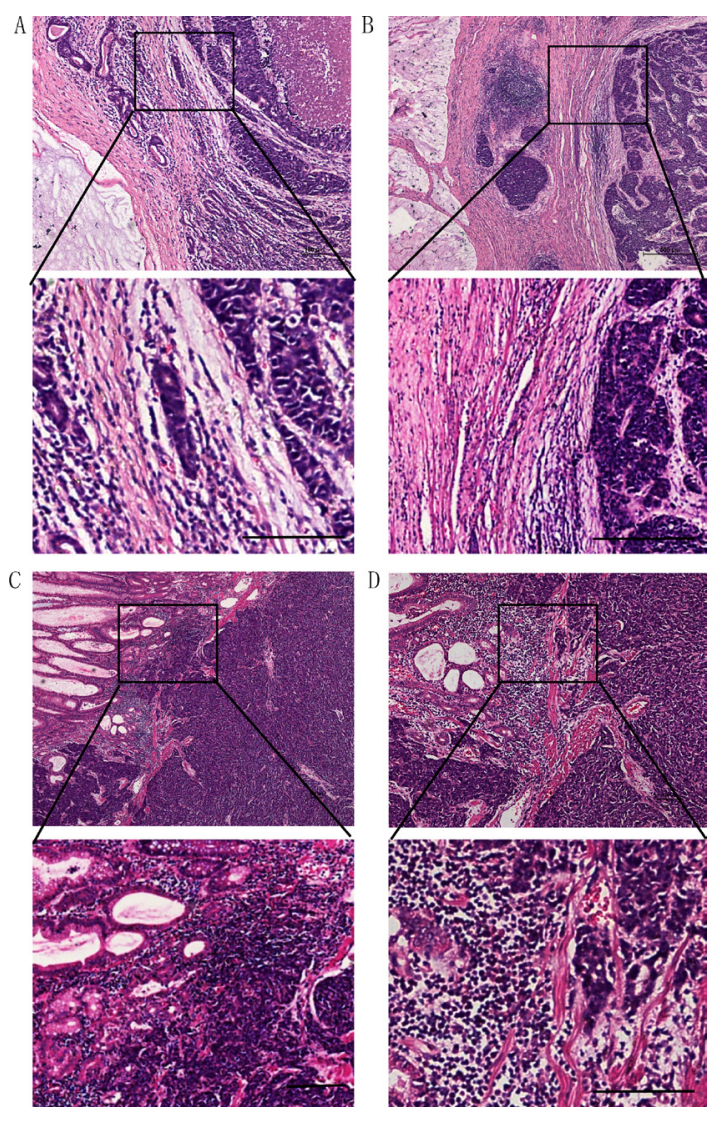

Figure 3: In gastric cardia NECs, some cases were mixed with mucinous adenocarcinoma, and with a clear border (A, B). Some were mixed with adenocarcinoma, and with a broken boundary $(\mathbf{C}, \mathbf{D})$.(Scale bar: $100 \mu \mathrm{m})$ 
is very useful for identifying typical NEC histology and cell differentiation. Positive staining of neuroendocrine markers is an important supplement for the diagnosis of NEC. Generally, neuroendocrine biomarkers, such as Syn and CgA, are essential for the diagnosis of NECs. In our patient cohort, NEC tumors display $100 \%$ positive staining for Syn, and $53.6 \%$ positive staining for $\mathrm{CgA}$, which is lower than a previous report [13]. Our findings are consistent with Huang et al., who reported that Syn staining is positive in all esophageal NECs [19].

There are limited reports concerning the outcome of patients with esophageal NEC, owing to the rarity of the disease. However, the prognosis for NEC is poor, since there is a high likelihood for disease recurrence. In this study, we performed Cox univariate and multivariate analysis and showed that tumor invasion and lymph node metastasis are independent factors for prognosis of NEC. The 1-and 3-year survival for esophageal NEC in our cases is 88.2 and $55.9 \%$, respectively. The poor prognosis of esophageal NECs could be a result of early lymphatic spreading and distant metastasis. Some have reported that invasion depth is the key factor for determining the survival of NEC patients [20]. The major difference of patient prognosis is between $\mathrm{T} 1$ and $\mathrm{T} 2-4$, indicating that patients have poor prognosis once the tumor has invaded the esophageal muscular layer. Early diagnosis and treatment are also important in that surgery has a limited effect, although joint postoperative adjuvant radiation and chemotherapy can obviously improve prognosis. Survival analyses by Lu et al. [21] and Ding et al. [22] reveal that joint treatment of patients is better than surgery only. Patients with mixed tumors have better prognosis than patients with pure NEC. However, there is no difference in prognosis between the large cell type and small cell type. A previous report stated that the prognosis of mixed NEC patients is worse compared with patients with pure NECs, but other reports indicate that the survival of mixed NEC patients and pure NEC is not obviously different [23].

In our study, we found that gastric cardia NECs are mostly mixed with high grade adenocarcinoma, and the borders are surrounded by loose connective tissue with inflammatory cells. Reports of gastric cardia NEC are very limited because of the unique physiological role of this mucosa. Some researchers found that neuroendocrine cells of gastric cardia are present in Barrett's esophagus, a severe gastro-esophageal reflux disease [24]. Voutilainen et al. reported $18 \mathrm{CgA}$-positive gastric cardia cases. However, they did not state the stage of the NETs. Most NETs in gastric cardia are of low-intermediate grade, and that among 76 cases of gastric cardia with NETs, only 11 cases involved NEC, which had a larger tumor size and deeper invasion [8].

Our paper studies 80 cases of esophageal and gastric cardia NEC, in the Chaoshan area, and characterizes the clinicopathological and immunohistochemical features, which may provide some therapeutic clue for treating this type of rare tumor.
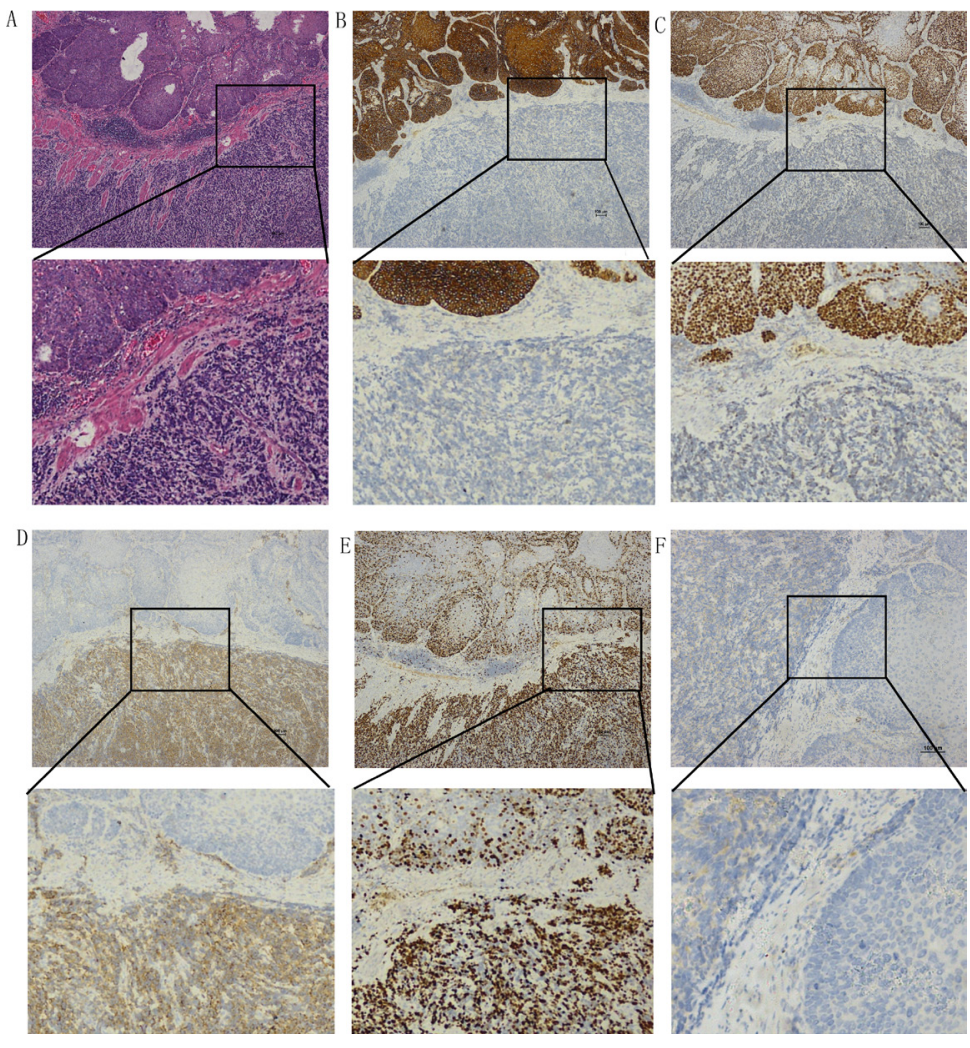

Figure 4: Examples of immunohistochemical staining of mixed type NEC and squamous carcinoma cells. (A) Hematoxylin-eosin stain. Immunoactivity to CK (B), P63 (C), Syn (D), Ki67 (E), and CgA (F).(Scale bar: $100 \mu \mathrm{m}$ ) 


\section{MATERIALS AND METHODS}

\section{Patient sample collection}

We collected and reviewed cases of patients diagnosed with primary esophageal and gastric cardia NEC from 2007 to 2016 in the Chaoshan area. The electronic medical records of all patients with esophageal and gastric cardia cancer, including the gender, age, tumor location, size, gross appearance, and histologic features, were searched in the Department of Pathology of the Shantou Tumor Hospital, First Affiliated Hospital of Shantou University Medical College and Shantou Central Hospital. All cases were independently reviewed and diagnosed by two pathologists. The study was approved by the ethical review committees of the Medical College of Shantou University. All participants involved in our study gave written informed consent. Retrospective collection of NECs had been approved by each ethical review committee of all participating centers. All experiments performed in this paper were in accordance with the relevant guidelines and regulations [1]

The inclusion criteria of case selection were as follows: 1. diagnosis of the pathology, along with the medical, radiologic, and endoscopic records, confirmed NEC of the esophagus and gastric cardia; 2. all clinical parameters, including the age, gender, and symptoms, were detailed in the records; 3 . neoplastic cells in the cases were immunoreactive to the WHO-recommended neuroendocrine markers, such as CD56, synaptophysin (Syn), and chromogranin A ( $\mathrm{CgA}) ; 4$. the center of the esophageal NEC tumor was located within $2 \mathrm{~cm}$ above the gastroesophageal junction (GEJ), defined by the American Joint Cancer Committee, eighth edition, for esophageal cancer, and the center of gastric cardia NEC tumors was located $>2 \mathrm{~cm}$ from the GEJ.

Exclusion criteria included the following: 1. the patient had prior chemotherapy or radiation treatment before surgical resection; 2. clinical data were not available, even if the cancer was diagnosed asesophageal or gastric cardia NEC; 3 . the patient had a history ofneuroendocrine carcinoma elsewhere.

\section{Histopathologic assessment}

NEC patient age, gender, symptoms, location, gross appearance, tumor size, clinical stage, classification, invasion, and lymph node metastasis, immunohistochemical expression, lymphovascular invasion, perineural invasion, radiologic findings, types of treatment and response to treatment were all collected. The location of the esophageal NEC was dividedinto three parts: upper (15-24 $\mathrm{cm}$ from the incisorteeth), middle (25-32 cm from the incisor teeth) and lower (33-40 cm from the incisor teeth). The location of gastric cardia NEC was a narrow band between the lower esophagus and gastric fundus that was limited to within $2 \mathrm{~cm}$ from the GEJ. Tumors located between the distal esophagus and gastric cardia were carefully evaluated based on the location and the adjacent mucosa. The gross appearance of esophageal and gastric cardia NEC was mainly categorized into 4 types: ulcerated, fungating, medullar and protuberant. Regional lymph nodeor distant metastasis was mainly evaluated using imagingmodalities, including CT and endoscopic ultrasonography (EUS), as well asother modalities. Lymph nodemetastasis was confirmed by apost-operative pathologic report if the imageological examination was uncertain. The locations of lymph node metastasis were also recorded. Results of lymphovascular invasion, perineural invasion and lymph nodemetastasis were presented as either positive or negative. The clinical stage of NECs followed the rules for ESCC as defined by the American Joint Cancer Committee in the eighth edition of the esophageal cancer staging manual, since standardized staging guidelines for NEC are lacking.

NEC was classified as small cell NEC (SNEC) and large cell NEC (LNEC) according to the WHO criteria for NEC [12]. SNEC cells are 2-3 times larger than lymphocytes, with scant cytoplasm and an inconspicuous nucleolus. However, LNEC cells are more than 4 times larger than lymphocytes, with ample cytoplasm and an evident nucleolus. Both SNEC and LNEC have and frequent necrosis and high mitotic rate $(>20 /$ high power field), with positive immunostaining for neuroendocrine markers. NEC can also be classified into 3 groups based on differentiation according to the 2000 WHO classificationcriteria: well-differentiated, moderatelydifferentiated and poorly-differentiated NEC.

\section{Immunohistochemistry and evaluation}

Immunohistochemical staining was performed using routine methods [13]. Images were captured using a Leica IM50 microscope (Imagic Bildverarbeitung AG, Wetzlar, Germany) at $\times 100, \times 200$ and $\times 400$. IHC staining was examined by two pathologists, who were blinded to the clinical outcome. Tumors were defined as positive if over $30 \%$ of neoplastic cells stained positively for the antigen. Staining was determined by consensus andthe discrepancy between readers was minimal.

Primary antibodies, CD56 (1:200),Syn (1:200), CgA (1:200), cytokeratin 5/6 (CK 5/6) (1:200), p63 (1:100),TTF-1 (1:200), smooth muscle actin (SMA) (1:200), Ki67 (1:500) and S-100 (1:200) were purchased from Maixin (China). Immunohistochemical staining results were presented as either positive or negative.

\section{Survival outcome}

We performed the final follow-up by telephone, mail or outpatient department visit in October 2016. 
Overall survival was calculated from the day of esophagogastroduodenoscopy and biopsy to thedate of death or last follow-up (months). Clinical follow-up data was available for 34/67 esophageal NEC patients and 3/13 of gastric cardia NEC patients included in this study.

\section{Statistical analysis}

Statistical analysis was performed using SPSS software (SPSS Corp, Chicago, IL, USA). Data are represented asthe mean \pm standard deviation for continuous variables or number (\%) for categorical data. To estimate the association between eligible variables and mean survival time,Kaplan-Meier analysis was applied. A $p$-value less than 0.05 was considered statistically significant.

\section{Ethics approval and consent to participate}

The study was approved by the ethical review committees of the Medical College of Shantou University. The patients involved in our study provided written informed consents.

\section{Consent for publication}

We received permission from the patients to publish the data.

\section{Author contributions}

ZYL collected the images and all the clinical parameters, LLH collected all the pathology reports and took pictures and $\mathrm{YZ}$ wrote the paper.

\section{ACKNOWLEDGMENTS}

We appreciate Stanley Lin revising the language and proposing some suggestions to improve this paper.

\section{CONFLICTS OF INTEREST} interest.

The authors declare that they have no conflicts

\section{FUNDING}

None.

\section{REFERENCES}

1. Ramage JK, Ahmed A, Ardill J, Bax N, Breen DJ, Caplin ME, Corrie P, Davar J, Davies AH, Lewington V, Meyer T, Newell-Price J, Poston G, et al. UK and Ireland Neuroendocrine Tumour Society. Guidelines for the management of gastroenteropancreatic neuroendocrine (including carcinoid) tumours (NETs). Gut. 2012; 61:6-32.

2. Bosman TF, Carniero F, Hruban RH, Theise ND, editors. The International Agency for Research on Cancer. WHO Classification of Tumours of the Digestive System. 4th ed. (Eds.). Lyon: IARC Press. 2010.

3. Plckinger U, Rindi G, Arnold R, Eriksson B, Krenning EP, de Herder WW, Goede A, Caplin M, Oberg K, Reubi JC, Nilsson O, Delle Fave G, Ruszniewski P, et al. European Neuroendocrine Tumour Society. Guidelines for the diagnosis and treatment of neuroendocrine gastrointestinal tumours. A consensus statement on behalf of the European Neuroendocrine Tumour Society (ENETS). Neuroendocrinology. 2004; 80:394-424.

4. Collado-Mesa F, Net JM, Klevos GA, Yepes MM. Primary neuroendocrine carcinoma of the breast: report of 2 cases and literature review. Radiol Case Rep. 2017; 12:1-12.

5. Sakuma Y, Yasuda Y, Sata N, Hosoya Y, Shimizu A, Fujii H, Matsubara D, Fukushima N, Miki A, Maeno M, Lefor AK. Pancreatic neuroendocrine tumor with metastasis to the spleen: a case report. BMC Cancer. 2017; 17:37.

6. Fabbri A, Cossa M, Sonzogni A, Papotti M, Righi L, Gatti G, Maisonneuve P, Valeri B, Pastorino U, Pelosi G. Ki-67 labeling index of neuroendocrine tumors of the lung has a high level of correspondence between biopsy samples and surgical specimens when strict counting guidelines are applied. Virchows Arch. 2017; 470:153-164.

7. Zhang Y, Wang H, Bi C, Xiao Y, Liu Z. Expression of CDX2 in gastric cardia adenocarcinoma and its correlation with $\mathrm{H}$. pylori and cell proliferation. Oncotarget. 2016; 7:54973-54982. https://doi.org/10.18632/oncotarget.10362.

8. Voutilainen M, Juhola M, Pitkänen R, Färkkilä M, Sipponen P. Immunohistochemical study of neuroendocrine cells at the gastric cardia mucosa. J Clin Pathol. 2002; 55:767-9.

9. Öberg K. Gastric neuroendocrine cells and secretory products. Yale J Biol Med. 1998; 71:149-54.

10. Wang Y, Liu S, Zhang Y, Bi C, Xiao Y, Lin R, Huang B, Tian $\mathrm{D}$, Ying $\mathrm{S}, \mathrm{Su} \mathrm{M}$. Helicobacter pylori infection and gastric cardia cancer in Chaoshan region. Microbes Infect. 2014; 16:840-4.

11. Tan HZ, Lin WJ, Huang JQ, Dai M, Fu JH, Huang QH, Chen WM, Xu YL, Ye TT, Lin ZY, Lin XS, Cai JX, Dong $\mathrm{YH}$, et al. Updated incidence rates and risk factors of esophageal cancer in Nan'ao Island, a coastal high-risk area in southern China. Dis Esophagus. 2016; 30:1-7.

12. Brierley JD, Gospodarowicz MK, Wittekind C. TNM Classification of Malignant Tumours, 8th Edition. ISBN: 978-1-119-26357-9P57.

13. Huang Q, Wu H, Nie L, Shi J, Lebenthal A, Chen J, Sun Q, Yang J, Huang L, Ye Q. Primary high-grade neuroendocrine carcinoma of the esophagus: a clinicopathologic and immunohistochemical study of 42 resection cases. Am J Surg Pathol. 2013; 37:467-83. 
14. Huang Q, Fang DC, Yu CG, Zhang J, Chen MH. Barrett's esophagus-related diseases remain uncommon in China. J Dig Dis. 2011; 12:420-7.

15. Nekulova M, Holcakova J, Coates P, Vojtesek B. The role of p63 in cancer, stem cells and cancer stem cells. Cell Mol Biol Lett. 2011; 16:296-327.

16. Maru DM, Khurana H, Rashid A, Correa AM, Anandasabapathy S, Krishnan S, Komaki R, Ajani JA, Swisher SG, Hofstetter WL. Retrospective study of clinicopathologic features and prognosis of high-grade neuroendocrine carcinoma of the esophagus. Am J Surg Pathol. 2008; 32:1404-11.

17. Nayal B, Vasudevan G, Rao AC, Kudva R, Valliathan M, Mathew M, Rao L. Primary Small Cell Carcinoma of The Esophagus - An Eight Year Retrospective Study. J Clin Diagn Res. 2015; 9:EC04-6.

18. Brenner B, Shah MA, Gonen M, Klimstra DS, Shia J, Kelsen DP. Small-cell carcinoma of the gastrointestinal tract: a retrospective study of 64 cases. Br J Cancer. 2004; 90:1720-6.

19. Huang Q, Shi J, Sun Q, Fan X, Feng A, Wu A, Zhou Q, Yu C, Mashimo H, Lauwers GY. Distal esophageal carcinomas in Chinese patients vary widely in histopathology, but adenocarcinomas remain rare. Hum Pathol. 2012; 43:21382148.

20. Gu XS, Zhou Y, Wang W, Luo JH, Chen L. Clinical significance of 41 cases of esophageal neuroendocrine carcinoma. Chin J Clin Thorac Cardiovasc Surg. 2016; 23:978-981.

21. Lu XJ, Luo JD, Ling Y, Kong YZ, Feng LL, Zhou J, Wang F. Management of small cell carcinoma of esophagus in China. J Gastrointest Surg. 2013; 17:1181-1187.

22. Ding J, Ji J, Zhu W, Han J, Zhang Y, Yu C, Li T, Tao G, Ji F, Zhou X, Pan P. A retrospective study of different treatments of limited-stage small-cell esophageal carcinoma and associated prognostic factor analysis. Dis Esophagus. 2013; 26:696-702.

23. Griffin M, Sweeney EC. The relationship of endocrine cells, dysplasia and carcinoembryonic antigen in Barrett's mucosa to adenocarcinoma of the oesophagus. Histopathology. 1987; 11:53-62.

24. Zhang N, Zhang SW, Wang Y, Li BQ. The two case of gastric cardia neuroendocrine carcinoma. Esophageal surgery magazine. 2014; 2:39-40. 